\title{
Reinforcing Polyamide 1212 with Oxidized Carbon Nanotubes
}

\author{
Ziqing Cai \\ Department of Materials, College of Science, China \\ University of Petroleum \\ Beijing 102249, China \\ E-mail: zqcaicup@gmail.com
}

\author{
Xiaoyu Meng* \\ Department of Materials, College of Science, China \\ University of Petroleum \\ Beijing 102249, China \\ E-mail: xymeng800418@,sohu.com
}

\author{
Lishan Cui \\ Department of Materials, College of Science, China \\ University of Petroleum \\ Beijing 102249, China \\ E-mail: lishancui63@126.com \\ Qiong Zhou* \\ Department of Materials, College of Science, China \\ University of Petroleum \\ Beijing 102249, China \\ E-mail: cuppolymer@163.com
}

\begin{abstract}
The polymer composites based on carbon nanotubes are of particular interests because of their excellent performance. In this work, carbon nanotubes (CNTs) were modified by concentrated acid solution which resulted in CNTs grafted with carboxylic acid $(-\mathrm{COOH})$ and hydroxyl (-OH) groups (i.e. a-CNTs). The polyamide 1212 (PA1212)/a-CNTs composites with a series of CNT contents were prepared by melt-compounding process. The mechanical properties of the PA1212/a-CNTs composites were reinforced efficiently with the addition of a-CNTs. The tensile strength, Young's modulus and yield strength of the composites were improved compared with these of pure PA1212. The dynamic modulus and $T_{g}$ of the composites were improved with the addition of a-CNTs. And it indicated that there existed obvious interfacial adhesion between CNTs and PA1212 matrix. The melt point, crystallization temperatures and crystallinities of the composites were improved due to the heterogeneous nucleation effect of aCNTs in PA1212 matrix. The a-CNTs induced the formation of $\gamma$-crystal form in PA1212 composites from WAXD results.
\end{abstract}

Keywords-Polyamide 1212; carbon nanotubes; mechanical properties; composites; reinforcement.

\section{INTRODUCTION}

Carbon nanotubes are widely used in the reinforcement of semi-crystalline polymers based on their excellent properties, such as high mechanical strength/modulus [1], large specific surface area/aspect ratio [2] and so on. However, reinforcing effect of nanofillers is not significant as expected [3]. The restraining factors include inadequate dispersion and alignment of the nanofillers, poor adhesion and load transfer at interfaces [3]. To improve the dispersion state and interfacial adhesion, the surface modification of CNTs is essential [4-5]. Meng et al. [6] prepared PA6/MWNTs composites with acid- and diamine-modified MWNTs by a simple melt compounding method. Better dispersion and stronger interfacial adhesion of modified MWNTs in PA6 matrix could be obtained. Mechanical properties of the composites were improved significantly with the addition of the modified MWNTs.
In this study, CNTs were modified by acid oxidation solution. Polyamide 1212 was used as the matrix materials and PA1212/a-CNTs composites with different CNTs contents were prepared by melt compounding. The effects of a-CNTs on mechanical properties and crystallization properties were investigated.

\section{EXPERIMENTAL SECTION}

PA1212 pellets $(\mathrm{MI}=3.75 \mathrm{~g} / 10 \mathrm{~min})$ were purchased from Shandong Dongcheng Engineering Plastic Co. Ltd. Pristine CNTs with average diameter of $11 \mathrm{~nm}$ and length of $10 \mu \mathrm{m}$ (>95\% purity) were purchased from Beijing Tiannai Nanotechnology Co.

Pristine CNTs were dispersed in concentrated $\mathrm{H}_{2} \mathrm{SO}_{4} / \mathrm{HNO}_{3}(3: 1 \mathrm{v} / \mathrm{v})$ solution in an ultrasonic bath $(40$ $\mathrm{kHz}, 200 \mathrm{~W}$ ) for $30 \mathrm{~min}$, and then mechanically stirred at $60{ }^{\circ} \mathrm{C}$ for $4 \mathrm{~h}$. The dispersion solution was then diluted with large quantity of water and washed with deionized water for several times until the $\mathrm{pH}$ of the solution reached 7.0. The CNTs were dried at $80{ }^{\circ} \mathrm{C}$ in a vacuum environment for $24 \mathrm{~h}$, resulting in CNTs grafted with carboxylic acid $(-\mathrm{COOH})$ and hydroxyl $(-\mathrm{OH})$ groups (aCNTs). The morphology of pristine CNTs and a-CNTs were characterized by scanning electron microscopy (SEM).

\section{A. Preparation of PA1212/CNTs composites}

Before melt blending, PA1212 pellets and a-CNTs were dried at $80{ }^{\circ} \mathrm{C}$ for $24 \mathrm{~h}$ in a vacuum oven. Composites with varying a-CNT contents $(0.3$ wt. $\%, 0.7$ wt. $\%$, and $1.0 \mathrm{wt} . \%)$ were prepared by melt blending in a Brabender mixer $(40 \mathrm{rpm})$ at $205^{\circ} \mathrm{C}$ for $12 \mathrm{~min}$. Plate specimens (1.2 $\mathrm{mm}$ thickness) were prepared by compression molding at $210{ }^{\circ} \mathrm{C}$ for $10 \mathrm{~min}$. The drawing dumbbell specimens were cut by the cutting mold, and the mold size was $4 \mathrm{~mm}$ width, and $75 \mathrm{~mm}$ length.

\section{B. Performance testing and structural characterization}


The mechanical properties of the samples were determined using an electronic universal tensile testing machine. The tensile strength, Young's modulus and elongation at breakage point were measured at room temperature at $50 \mathrm{~mm} / \mathrm{min}$.

The thermal properties of the samples were determined by differential scanning calorimetry (DSC 204 F1, NETZSCH) under argon atmosphere. For non-isothermal melt crystallization, the samples were heated from $25^{\circ} \mathrm{C}$ to $220^{\circ} \mathrm{C}$ at $10^{\circ} \mathrm{C} / \mathrm{min}$ rate, held for $2 \mathrm{~min}$ to remove thermal history, and then cooled down to $25{ }^{\circ} \mathrm{C}$. The meltingcrystallization temperatures $\left(T_{m}\right.$ and $\left.T_{c}\right)$ and crystallinities $\left(X_{c}\right)$ were determined.

The dynamic mechanical properties were carried out in TA-Q800 dynamic mechanical analyzer (DMA) using a tension mode at $1 \mathrm{~Hz}$ with a temperature ramp of $3{ }^{\circ} \mathrm{C} / \mathrm{min}$. Storage modulus and loss factor were obtained from $-20^{\circ} \mathrm{C}$ to $180{ }^{\circ} \mathrm{C}$ for pure PA1212 and composites.

Wide-angle X-ray diffraction (WAXD) patterns were obtained using a D8 Focus diffractometer at room temperature, with a wavelength of $0.154 \mathrm{~nm}$ in a $2 \theta$ range of $5-45^{\circ}$ at $2 \% \mathrm{~min}$.

\section{RESULTS AND DISCUSSION}

A. Morphology of CNTs
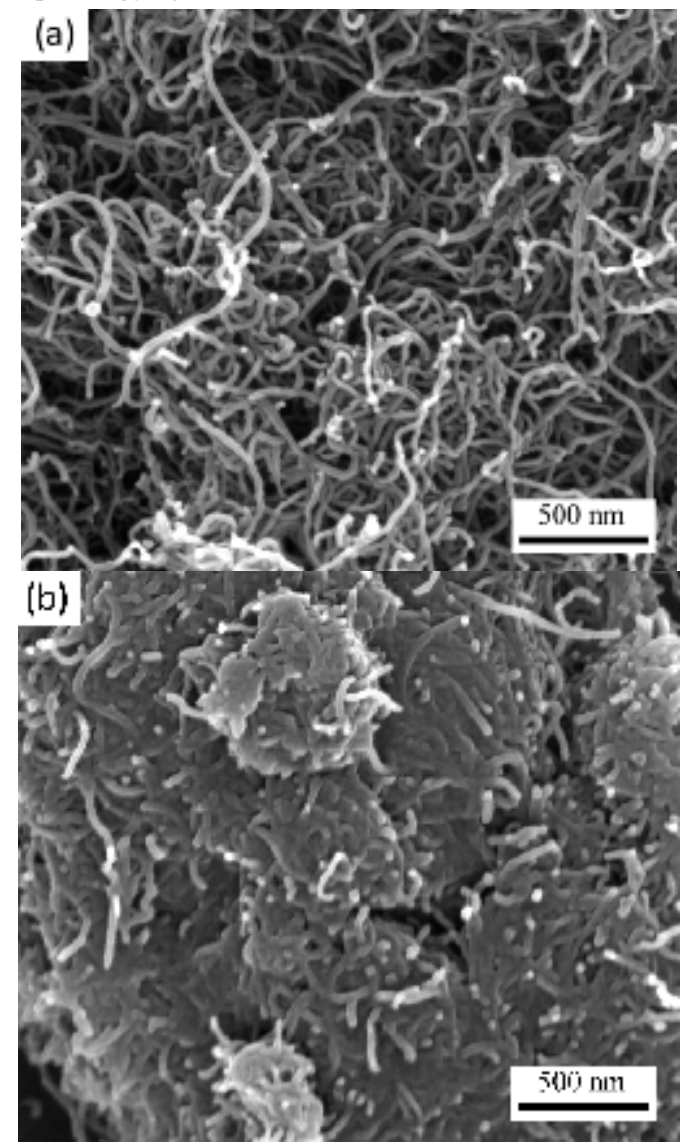

Figure 1. SEM images of pristine CNTs (a) and acid modified CNTs (a-CNTs) (b).

To improve the dispersion and interfacial adhesion of CNTs in PA1212 matrix, surface modification of CNTs is necessary. Given that $\mathrm{CNT}$ s have $\mathrm{C}-\mathrm{C}$ and $\mathrm{C}=\mathrm{C}$ structures on the surfaces, strong acid oxidation can be employed for surface modification. The functional groups including $\mathrm{COOH}$ and $-\mathrm{OH}$ can be introduced to the surface of CNTs.
The morphology of pristine CNTs and a-CNTs is shown in Fig. 1. From Fig. 1a, pristine CNTs exhibit smooth surface and they are randomly entangled together. From Fig. 1b, the morphology of a-CNTs is different; they exhibit rough surface and shorter length after modification, and aggregate together due to the functional groups on the surfaces. To some extent, these shorten CNTs are easy to disperse in PA1212 matrix.

\section{B. Mechanical properties of pure PA1212 and PA1212/a-CNTs composites}

The a-CNFs were used in preparation of the composites. The relationship between the mechanical properties and the a-CNT content is shown in Fig. 2 and Table 1. With addition of 0.3 wt. $\%$ a-CNTs, the yield strength and Young's modulus of the composite are improved from 45.6 $\mathrm{MPa}$ and $143.6 \mathrm{MPa}$ to $54.3 \mathrm{MPa}$ and 177.9 MPa, respectively. With the increase of CNT content, the Young's modulus of the composites is improved gradually. The tensile strength of composites decreases slightly due to the decrease of elongation at break. The reinforcing effect can be demonstrated by improvement of the yield strength and Young's modulus.

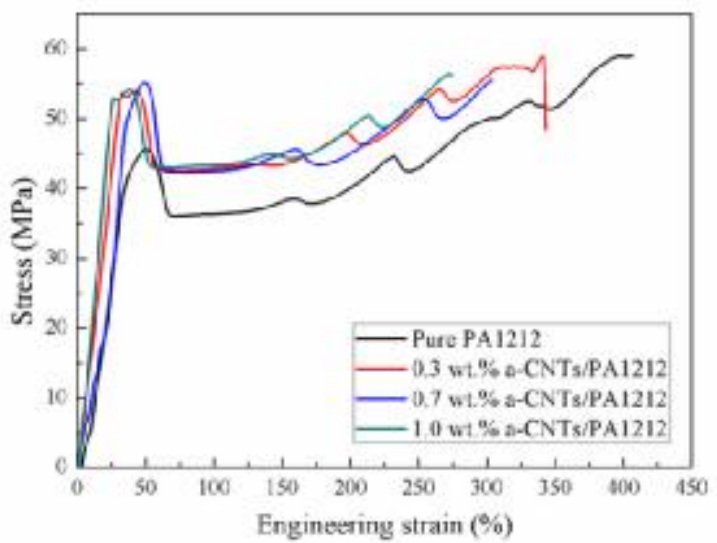

Figure 2. Mechanical properties as a function of a-CNTs content in PA1212 composites.

TABLE 1 The mechanical properties of pure PA1212 and the composites.

\begin{tabular}{ccccc}
\hline Samples & $\begin{array}{c}\text { Tensile } \\
\text { strength } \\
(\mathrm{MPa})\end{array}$ & $\begin{array}{c}\text { Yield } \\
\text { strength } \\
(\mathrm{MPa})\end{array}$ & $\begin{array}{c}\text { Young's } \\
\text { modulus } \\
(\mathrm{MPa})\end{array}$ & $\begin{array}{c}\text { Elongation } \\
\text { at break (\%) }\end{array}$ \\
\hline $\begin{array}{c}\text { Pure PA1212 } \\
\text { 0.3 wt.\% a- }\end{array}$ & $59.0 \pm 1.7$ & $45.6 \pm 1.5$ & $143.6 \pm 45.8$ & $405.0 \pm 18.8$ \\
CNTs/PA1212 & $59.0 \pm 1.1$ & $54.3 \pm 0.6$ & $177.9 \pm 9.0$ & $340.8 \pm 21.9$ \\
$\begin{array}{c}\text { 0.7 wt.\% a- } \\
\text { CNTs/PA1212 }\end{array}$ & $55.5 \pm 2.5$ & $55.2 \pm 0.3$ & $229.4 \pm 35.5$ & $303.1 \pm 20.1$ \\
$\begin{array}{c}\text { 1.0 wt.\% a- } \\
\text { CNTs/PA1212 }\end{array}$ & $56.4 \pm 1.1$ & $54.1 \pm 0.7$ & $230.8 \pm 16.9$ & $272.7 \pm 23.9$ \\
\hline
\end{tabular}



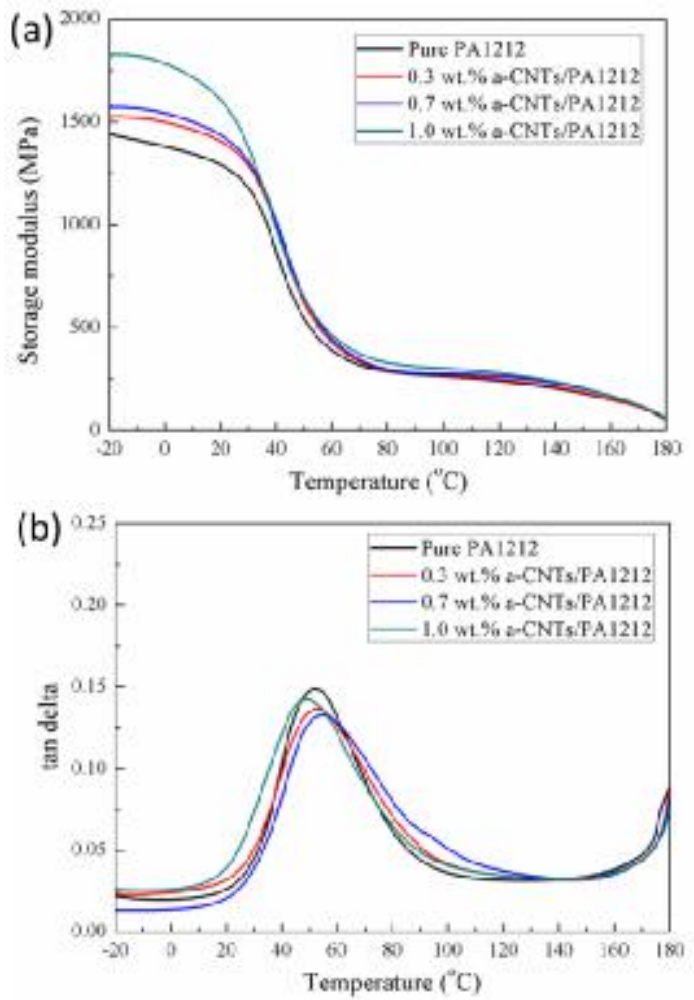

Figure 3. The storage modulus (a) and loss factor (b) of pure PA1212 and the composites as a function of temperature.

As shown in Fig. 3a, below glass transition temperature $\left(-20 \sim 50{ }^{\circ} \mathrm{C}\right)$, the storage modulus of PA1212 can be improved efficiently with the addition of a-CNTs and it indicates reinforcement effect of a-CNTs in PA1212 matrix. Especially for 1.0 wt. $\%$ PA1212/a-CNTs composite, the reinforcing effect is more obvious compared with that of the composites containing $0.3 \mathrm{wt} . \%$ and 0.7 wt.\% a-CNTs. Fig. $3 b$ shows the effect of a-CNTs on the loss factor in the composites. The peak values of the loss factor around $50{ }^{\circ} \mathrm{C}$ are assigned to the glass transition temperature $\left(T_{g}\right)$. The $T_{g}$ can effectively represent the mobility of polymer chains. As presented in Fig. 3b, the $T_{g}$ of pure PA1212 is about $52.0^{\circ} \mathrm{C}$, and $T_{g}$ of the composite is increased to $54.5^{\circ} \mathrm{C}$ as the a-CNTs content is increased to $0.7 \mathrm{wt} \%$. It indicates that the mobility of PA1212 molecule chains can be hindered with the addition of aCNTs and that resulted in improved $T_{g}$.

\section{Crystallization behavior of pure PA1212 and PA1212/a-CNTs composites}

The effect of CNTs on the crystallization behavior of PA1212/CNTs composites was analyzed by nonisothermal DSC experiments (Fig. 4 and Table 2). The crystallinity $\left(X_{c}\right)$ is determined as the ratio of the integrated heat of fusion value of the sample over the heat of fusion of purely crystalline PA1212,

$$
X_{c}=\frac{\Delta H_{m} \times 100}{\Delta H_{m}^{o}}
$$

where $\Delta H_{m}$ and $\Delta H_{m}^{o}$ are the enthalpy of fusion of samples and the equilibrium melting enthalpy, respectively.
The $\Delta H_{m}^{o}$ value for PA1212 is $292.2 \mathrm{~J} / \mathrm{g}$ [7]. The $X_{c}$ values calculated are shown in Table 2.
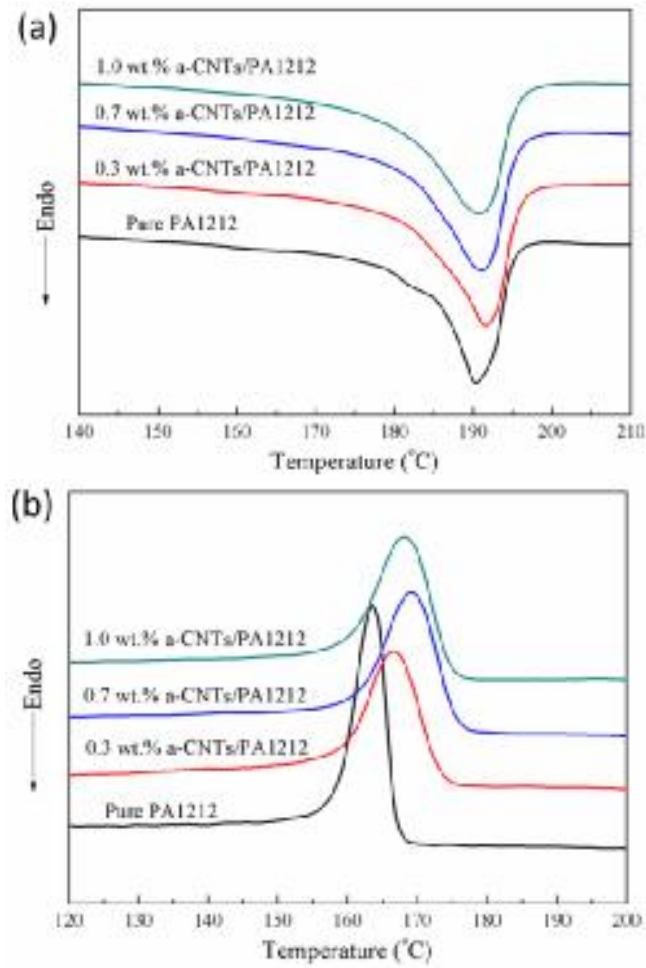

Figure 4. Non-isothermal DSC scans of pure PA1212 and PA1212/a-CNTs composites: (a) melting curves, (b) crystallization curves.

TABLE 2 DSC analysis of pure PA1212 and PA1212/aCNTs composites.

\begin{tabular}{ccccc} 
Samples & $T_{m}\left({ }^{\circ} \mathrm{C}\right)$ & $T_{c, o}\left({ }^{\circ} \mathrm{C}\right)$ & $T_{c, p}\left({ }^{\circ} \mathrm{C}\right)$ & $X_{c}(\%)$ \\
\hline Pure PA1212 & 190.3 & 166.9 & 163.6 & 12.4 \\
0.3 wt.\% a-CNTs/PA1212 & 191.6 & 173.2 & 166.6 & 14.9 \\
0.7 wt.\% a-CNTs/PA1212 & 191.1 & 175.4 & 169.1 & 15.2 \\
1.0 wt.\% a-CNTs/PA1212 & 190.9 & 174.5 & 168.2 & 17.2 \\
\hline
\end{tabular}

As shown in Fig. 4b, a-CNTs show efficient heterogeneous nucleation effect during crystallization of the composites. With the addition of $0.3 \mathrm{wt} . \% \mathrm{a}-\mathrm{CNTs}$, the crystallization onset temperature $\left(T_{c, o}\right)$ and peak temperature $\left(T_{c, p}\right)$ increase by $\sim 6^{\circ} \mathrm{C}$ and $\sim 3^{\circ} \mathrm{C}$ respectively. When the CNT content is increased to $0.7 \mathrm{wt} . \%$, the maximum improvement of $T_{c, o}$ and $T_{c, p}$ are observed. The melting points of PA1212/a-CNTs composites are higher than that of pure PA1212 (from Table 2). Crystallinities of the composites are improved gradually with the increase of a-CNT content (from 0 to 1.0 wt.\%) due to the heterogeneous nucleation effect. 
D. WAXD analysis of pure PA1212 and PA1212/a-CNTs composites

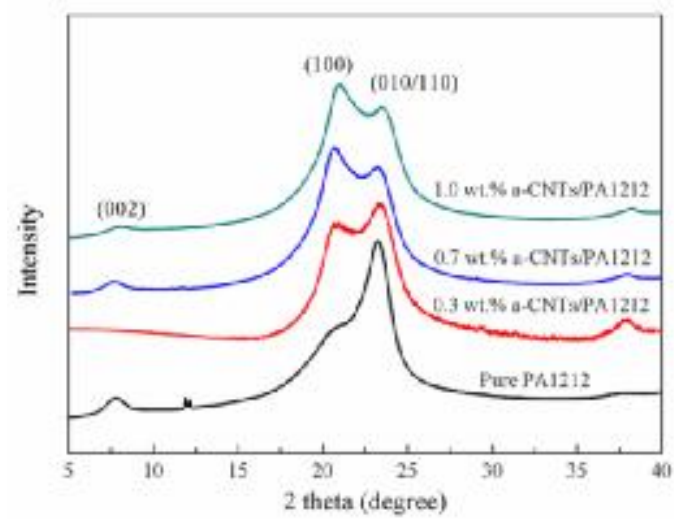

Figure 5. WAXD analysis of pure PA1212 and PA1212/aCNTs composites (thermal treatment at $100{ }^{\circ} \mathrm{C}$ for $12 \mathrm{~h}$ ).

The $\alpha$ - and $\gamma$-crystal forms can coexist in the same crystallite of PA1212 [7]. As shown in Fig. 5, two distinct peaks are observed in all of the samples; these peaks correspond to the (100) and (010/110) crystal planes. Thus, PA1212 crystallizes in the triclinic $\alpha$-form dominantly, and the $(010 / 110)$ crystal plane is the characteristic $\alpha$-crystal form. The (100) crystal plane is the characteristic $\gamma$-crystal form. The (010) crystal plane consists of sheets of hydrogen-bonded chains formed between parallel chains, and this plane has the priority growth [8]. With the addition of a-CNTs, the relative diffraction intensity of (100) crystal plane of the composites increases with respect to that of the $(010 / 110)$ crystal plane. The presence of a-CNTs facilitates the formation of less-ordered $\gamma$ crystal form. This result is consistent with the results of PA6/clay nanocomposites, in which the addition of nanoclay filler induced the formation of $\gamma$-crystal form [9-10].

\section{CONCLUSIONS}

CNTs were modified by $\mathrm{H}_{2} \mathrm{SO}_{4} / \mathrm{HNO}_{3}(3: 1 \mathrm{v} / \mathrm{v})$ solution which resulted in CNTs grafted with $-\mathrm{COOH} /$ $\mathrm{OH}$ groups (i.e. a-CNTs). The Young's modulus, yield strength and storage modulus of the PA1212/CNTs composites were reinforced efficiently with the addition of a-CNTs. For 0.7 wt.\% PA1212/a-CNTs composite, the $T_{g}$ of the composite was improved due to the interfacial interaction between a-CNTs and PA1212 matrix. The melt point, crystallization temperature and crystallinities of the composites were improved due to the heterogeneous nucleation effect of a-CNTs in PA1212 matrix. According to WAXD results, a-CNTs induced the formation of $\gamma$ crystal form in PA1212 composites.

\section{REFERENCES}

[1] G.G, Tibbetts, M.L Lake, K.L Strong, B.P. Rice. Compos. Sci. Technol. 2007, 67, 1709-1718.

[2] J.N. Coleman, U. Khan, Y.K. Gun'ko. Adv. Mater. 2006, 18, 689-706.

[3] Y. Dzenis. Science 2008, 319, 419-420.

[4] N.G. Sahoo, S. Rana, J.W. Cho, L. Li, S.H. Chan. Prog. Polym. Sci. 2010, 35, 837-867.

[5] Z.Q. Cai, X.Y. Meng, H.M. Ye, C.B. Cong, Y.F. Wang, L.S. Cui, Q. Zhou. Materials and Design 2014, 63, 691-698

[6] H. Meng, G.X. Sui, P.F. Fang, R.Yang. Polymer 2008, 49, 610-620.

[7] M.Q. Ren, Z.S. Mo, Q.Y. Chen, J.B. Song. Polymer 2004, 45, 3511-3518.

[8] Q.X. Zhang, Z.S. Mo, H.F. Zhang, S.Y. Liu. Polymer 2001, 42, 5543-5547.

[9] T.X. Liu, Z.H. Liu, K.X. Ma, L. Shen. Compos. Sci. Technol. 2003, $63,331-337$.

[10] D.M. Lincoln, R.A. Vaia. Macromolecules 2004, 37, 4554-4561. 